Supplement of Biogeosciences, 17, 4043-4057, 2020 https://doi.org/10.5194/bg-17-4043-2020-supplement (C) Author(s) 2020. CC BY 4.0 License.

(c) (i)

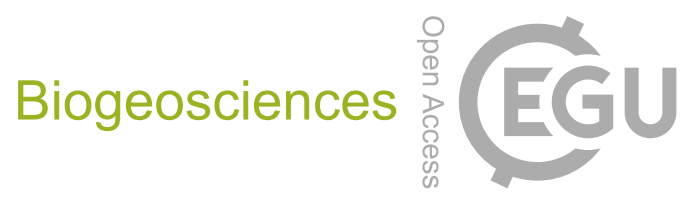

Supplement of

\title{
A Bayesian approach to evaluation of soil biogeochemical models
}

Hua W. Xie et al.

Correspondence to: Hua W. Xie (xiehw@uci.edu)

The copyright of individual parts of the supplement might differ from the article licence. 
Table S1: List of CON and AWB model parameters.

\begin{tabular}{|c|c|c|c|c|}
\hline$\underline{\text { Model }}$ & $\underline{\text { Parameter }}$ & Value, if not varied & $\underline{\text { Units }}$ & $\underline{\text { Parameter Description }}$ \\
\hline CON/AWB & $I_{S}$ & 0.0009 & $\mathrm{mg} \mathrm{C} \mathrm{g}^{-1}$ soil h$^{-1}$ & External SOC input rate \\
\hline CON/AWB & $I_{D}$ & 0.0001 & $\mathrm{mg} \mathrm{C} \mathrm{g}^{-1}$ soil $^{-1}$ & External DOC input rate \\
\hline $\mathrm{CON}$ & $k_{S_{r e f}}$ & Dependent & $\mathrm{mg} \mathrm{C} \mathrm{mg}{ }^{-1} \mathrm{C} \mathrm{h}^{-1}$ & SOC decay constant \\
\hline $\mathrm{CON}$ & $k_{D_{\text {ref }}}$ & Dependent & $\mathrm{mg} \mathrm{C} \mathrm{mg}{ }^{-1} \mathrm{C} \mathrm{h}^{-1}$ & DOC decay constant \\
\hline $\mathrm{CON}$ & $k_{M_{\text {ref }}}$ & Dependent & $\mathrm{mg} \mathrm{C} \mathrm{mg}{ }^{-1} \mathrm{C} \mathrm{h}^{-1}$ & MIC decay constant \\
\hline $\mathrm{CON}$ & $u_{M}$ & 0.002 & $\mathrm{mg} \mathrm{C} \mathrm{mg}{ }^{-1} \mathrm{C} \mathrm{h}^{-1}$ & DOC uptake rate of microbes \\
\hline $\mathrm{CON}$ & $E a_{S}$ & Fitted by HMC & $\mathrm{kJ} \mathrm{mol}^{-1}$ & SOC decomposition activation energy \\
\hline $\mathrm{CON}$ & $E a_{D}$ & Fitted by HMC & $\mathrm{kJ} \mathrm{mol}^{-1}$ & DOC decomposition activation energy \\
\hline $\mathrm{CON}$ & $E a_{M}$ & Fitted by HMC & $\mathrm{kJ} \mathrm{mol}^{-1}$ & MIC decomposition activation energy \\
\hline $\mathrm{CON}$ & $a_{D S}$ & Fitted by HMC & & DOC to SOC transfer coefficient \\
\hline $\mathrm{CON}$ & $a_{S D}$ & Fitted by HMC & & SOC to DOC transfer coefficient \\
\hline $\mathrm{CON}$ & $a_{M}$ & Fitted by HMC & & MIC to SOC transfer coefficient \\
\hline CON/AWB & $a_{M S}$ & Fitted by HMC & & Fraction of dead MIC transferred \\
\hline AWB & $K_{\text {ref }}$ & Dependent & $\mathrm{mg} \mathrm{C} \mathrm{g}^{-1}$ soil & SOC reference $K_{M}$ \\
\hline AWB & $K_{U_{r e f}}$ & Dependent & $\mathrm{mg} \mathrm{C} \mathrm{g}^{-1}$ soil & DOC uptake into MIC reference $K_{M}$ \\
\hline AWB & $V_{\text {ref }}$ & Fitted by HMC & $\mathrm{mg} \mathrm{C} \mathrm{mg}{ }^{-1} \mathrm{C} \mathrm{h}^{-1}$ & SOC reference $V_{\max }$ \\
\hline AWB & $V_{U_{r e f}}$ & Fitted by HMC & $\mathrm{mg} \mathrm{C} \mathrm{mg}{ }^{-1} \mathrm{C} \mathrm{h}^{-1}$ & DOC uptake into MIC reference $V_{\max }$ \\
\hline $\mathrm{AWB}$ & $E a_{K}$ & Fitted by HMC & $\mathrm{kJ} \mathrm{mol}^{-1}$ & SOC $K_{M}$ activation energy \\
\hline $\mathrm{AWB}$ & $E a_{K U}$ & Fitted by HMC & $\mathrm{kJ} \mathrm{mol}^{-1}$ & DOC uptake $K_{M}$ activation energy \\
\hline $\mathrm{AWB}$ & $E a_{V}$ & Fitted by HMC & $\mathrm{kJ} \mathrm{mol}^{-1}$ & SOC $V_{\max }$ activation energy \\
\hline AWB & $E a_{V U}$ & Fitted by HMC & $\mathrm{kJ} \mathrm{mol}^{-1}$ & DOC uptake $V_{\max }$ activation energy \\
\hline $\mathrm{AWB}$ & $r_{E}$ & Dependent & $\mathrm{mg} \mathrm{C} \mathrm{mg}{ }^{-1} \mathrm{C} \mathrm{h}^{-1}$ & Enzyme production rate \\
\hline AWB & $r_{L}$ & 0.0005 & $\mathrm{mg} \mathrm{C} \mathrm{mg}{ }^{-1} \mathrm{C} \mathrm{h}^{-1}$ & Enzyme loss rate \\
\hline $\mathrm{AWB}$ & $r_{M}$ & Dependent & $\mathrm{mg} \mathrm{C} \mathrm{mg}{ }^{-1} \mathrm{C} \mathrm{h}^{-1}$ & MIC death rate \\
\hline $\mathrm{AWB}$ & $E_{C_{r e f}}$ & Fitted by HMC & $\mathrm{mg} \mathrm{C} \mathrm{mg}^{-1} \mathrm{C}$ & Reference temperature $\mathrm{C}$ use efficiency (CUE) \\
\hline $\mathrm{AWB}$ & $m_{t}$ & Fitted by HMC & ${ }^{\circ} \mathrm{C}^{-1}$ & CUE temperature change slope \\
\hline
\end{tabular}




\section{Section S1}

(a) CON ODE system equations

18 The conventional (CON) model consists of three $\mathrm{C}$ pools in SOC, DOC, and MIC. The mass transfer of C between

$$
\frac{d S}{d t}=I_{S}+a_{D S} k_{D} D+a_{M} a_{M S} k_{M} M-k_{S} S
$$

where $R$ is the ideal gas constant $8.314 \mathrm{~J} \mathrm{~mol}^{-1} \mathrm{~K}^{-1}$ and the reference temperature $T_{\text {ref }}$ used was $283.15 \mathrm{~K}$.

The decay constants $k_{I}$ vary from their reference values $k_{\text {Iref }}$ based on the Arrhenius equation of temperature dependence,

$$
\frac{d D}{d t}=I_{D}+a_{S D} k_{S} S+a_{M}\left(1-a_{M S}\right) k_{M} M-u_{M} D-k_{D} D
$$

$$
\frac{d M}{d t}=u_{M} D-k_{M} M
$$

$34 \mathrm{CO}_{2}$ soil flux is calculated from the $\mathrm{CON}$ model by summing the proportion of fluxes that do not enter soil $\mathrm{C}$ pools at each time step:

$$
\text { CON flux }=k_{S} S\left(1-a_{S D}\right)+k_{D} D\left(1-a_{D S}\right)+k_{M} M\left(1-a_{M}\right)
$$

Response ratios are then calculated from the model output flux by dividing the flux calculated at a given time point by the pre-warming steady state flux.

42 (b) AWB ODE system equations

The Allison-Wallenstein-Bradford (AWB) model consists of four C pools in SOC, DOC, MIC, and ENZ

44 (representing the extracellular enzyme C mass). In the AWB model, MIC accumulation and SOC decomposition follow a non-linear Michaelis-Menten function. Other processes, including ENZ production, ENZ loss, and MIC death still follow a first-order linear decay process. The AWB system equations are as follows:

$$
\begin{gathered}
\frac{d S}{d t}=I_{S}+a_{M S} r_{M} M-\frac{V E S}{K+S} \\
\frac{d D}{d t}=I_{D}+\left(1-a_{M S}\right) r_{M} M+\frac{V E S}{K+S}+r_{L} E-\frac{V_{U} M D}{K_{U}+D} \\
\frac{d M}{d t}=E_{C} \frac{V_{U} M D}{K_{U}+D}-r_{M} M-r_{E} M \\
\frac{d E}{d t}=r_{E} M-r_{L} E
\end{gathered}
$$

56 Similar to the CON decay constants, the Michaelis-Menten function parameters $K, K_{U}, V$, and $V_{U}$ vary from their reference values based on the Arrhenius equation. $E_{C}$, the AWB microbial $C$ use efficiency parameter, depends linearly on temperature, following $\mathrm{Li}$ et al., 2014, and operates under the simplifying assumption that higher temperatures make $\mathrm{C}$ use slightly less efficient:

$$
E_{C}=E_{C_{r e f}}-m_{t}\left(T-T_{r e f}\right)
$$


The loss rate parameters $r_{I}$ were not made to be temperature dependent.

AWB $\mathrm{CO}_{2}$ flux is calculated as the proportion of the $\mathrm{C}$ transfer out of the DOC pool that is not partitioned into the 66 MIC pool:

68

$$
\text { AWB flux }=\left(1-E_{C}\right) \frac{V_{U} M D}{K_{U}+D}
$$

70

72

74

76

78

80

82

84

86

88

90

92

94

96

98

100

102

104

106

108

110

112

114

116 


\section{Section S2}

(a) Re-arranged CON steady state equations

The steady state solutions for the $\mathrm{C}$ pools in $\mathrm{CON}$ are as follows:

$$
\begin{gathered}
D_{0}=\frac{a_{S D} I_{S}+I_{D}}{u_{M}+k_{D}+u_{M} a_{M}\left(a_{M S}-a_{M S} a_{S D}-1\right)-a_{D S} k_{D} a_{S D}} \\
M_{0}=\frac{u_{M}}{k_{M}} D_{0} \\
S_{0}=\frac{I_{S}+D_{0}\left(a_{D S} k_{D}+u_{M} a_{M} a_{M S}\right)}{k_{S}}
\end{gathered}
$$

To set pre-warming steady state soil $\mathrm{C}$ densities to desired values, we re-arranged the steady state equations into the following forms to solve for the steady state values of parameters that depend on the soil C densities:

$$
k_{\text {Mref }}=\frac{u_{M} D_{0}}{M_{0}}
$$

$$
k_{\text {Dref }}=\frac{-I_{D}-a_{S D} I_{S}+u_{M} D_{0}-a_{M} D_{0} u_{M}+a_{M} a_{M S} u_{M} D_{0}-a_{M} a_{M S} a_{S D} u_{M} D_{0}}{\left(a_{D S} a_{S D}-1\right) D_{0}}
$$

$$
k_{\text {Sref }}=\frac{I_{S}+D_{0}\left(a_{D S} k_{\text {Dref }}+u_{M} a_{M} a_{M S}\right)}{S_{0}}
$$

(b) Re-arranged AWB steady state equations

138 The steady state solutions for the C pools in AWB are as follows:

$$
S_{0}=\frac{-r_{L} K\left(I_{S}\left(r_{M}\left(1+E_{C}\left(a_{M S}-1\right)\right)+r_{E}\left(1-E_{C}\right)\right)+E_{C} I_{D} a_{M S} r_{M}\right)}{I_{S}\left(r_{M}\left(r_{L}\left(1+E_{C}\left(a_{M S}-1\right)\right)\right)+r_{E}\left(r_{L}\left(1-E_{C}\right)-E_{C} V\right)\right)+E_{C} I_{D}\left(a_{M S} r_{M} r_{L}-r_{E} V\right)}
$$

$$
M_{0}=\frac{E_{C}\left(I_{D}+I_{S}\right)}{\left(1-E_{C}\right)\left(r_{M}+r_{E}\right)}
$$

$$
D_{0}=\frac{-K_{U}\left(r_{M}+r_{E}\right)}{r_{M}+r_{E}-E_{C} V_{U}}
$$

$$
E_{0}=\frac{r_{E} M_{0}}{r_{L}}
$$

To set pre-warming steady state soil $\mathrm{C}$ densities to desired values, we re-arranged the steady state equations into the following forms:

$$
\begin{gathered}
r_{E}=\frac{r_{L} E_{0}}{M_{0}} \\
r_{\mathrm{M}}=\frac{-E_{C_{r e f}\left(I_{D}+I_{S}\right)+M_{0} r_{E}\left(1-E_{C_{r e f}}\right)}}{M_{0}\left(E_{C_{r e f}}-1\right)}
\end{gathered}
$$

$$
K_{U_{\text {ref }}}=\frac{-D_{0}\left(r_{M}+r_{E}-E_{C_{r e f}} V_{U_{r e f}}\right)}{r_{M}+r_{E}}
$$




$$
K_{r e f}=\frac{-S_{0}\left(-I_{S} r_{E} r_{L}+E_{C_{r e f}} I_{S} r_{E} r_{L}-a_{M S} E_{C_{r e f}} I_{D} r_{L} r_{M}-I_{S} r_{L} r_{M}+E_{C_{r e f}} I_{S} r_{L} r_{M}-a_{M S} E_{C_{r e f}} I_{S} r_{L} r_{M}+E_{C_{r e f}} I_{D} r_{E} V_{r e f}+E_{C_{r e f}} I_{S} r_{E} V_{r e f}\right)}{r_{L}\left(-I_{S} r_{E}+E_{C_{r e f}} I_{S} r_{E}-a_{M S} E_{c_{r e f}} I_{D} r_{M}-I_{S} r_{M}+E_{C_{r e f}} I_{S} r_{M}-a_{M S} E_{c_{r e f}} I_{S} r_{M}\right)}
$$

156

158

160

162

164

166

168

170

172

174

176

178

180

182

184

186

188

190

192

194

196

198

200

202

204

206 
Figure S1: Distribution of CON and AWB fits to meta-analysis data (Romero-Olivares et al., 2017) with CON fits at (a) $\mathrm{MIC}=1 \mathrm{mg} \mathrm{C} \mathrm{g}^{-1}$ soil; and (b) $\mathrm{MIC}=8 \mathrm{mg} \mathrm{C} \mathrm{g}^{-1}$ soil.; and AWB fits at (c) $\mathrm{MIC}=1 \mathrm{mg} \mathrm{C} \mathrm{g}^{-1}$ soil; and (d) $\mathrm{MIC}=8 \mathrm{mg} \mathrm{C} \mathrm{g}^{-1}$ soil. Open circles show the meta-analysis data points. Blue vertical lines mark the $95 \%$ confidence interval for each data point calculated from the pooled standard deviation. The black line indicates the mean posterior predictive model fit. The orange shading marks the $95 \%$ posterior predictive interval for the fit. NonMIC pre-warming steady state soil C densities were set at $\mathrm{SOC}=100 \mathrm{mg} \mathrm{C} \mathrm{g}^{-1}$ soil, $\mathrm{DOC}=0.2 \mathrm{mg} \mathrm{C} \mathrm{g}^{-1}$ soil, and $214 \quad \mathrm{ENZ}=0.1 \mathrm{mg} \mathrm{C} \mathrm{g}^{-1}$ soil.

(a)

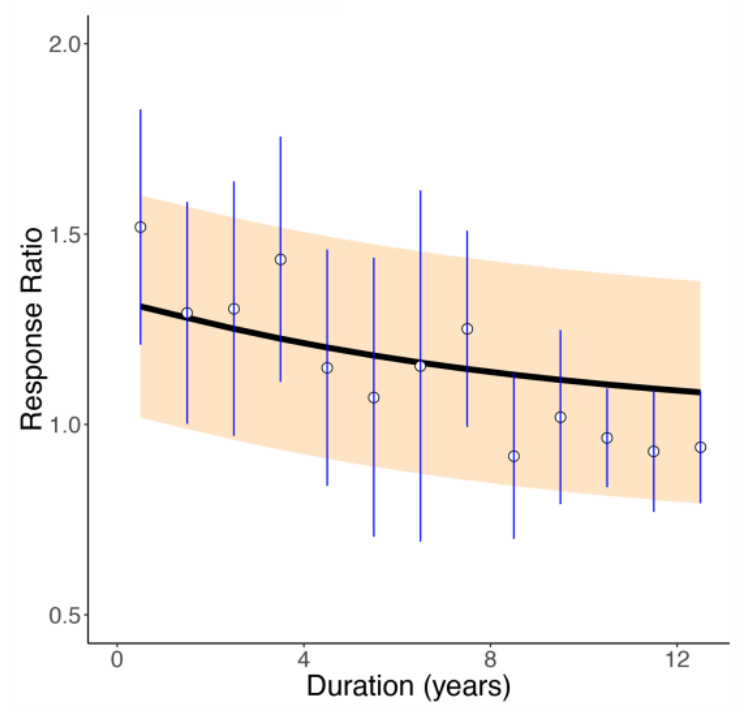

(c)

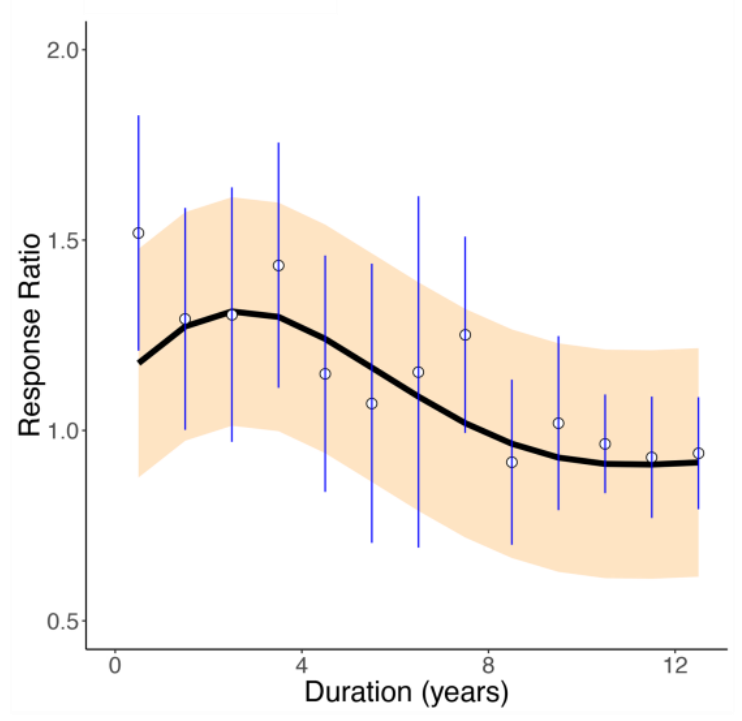

(b)

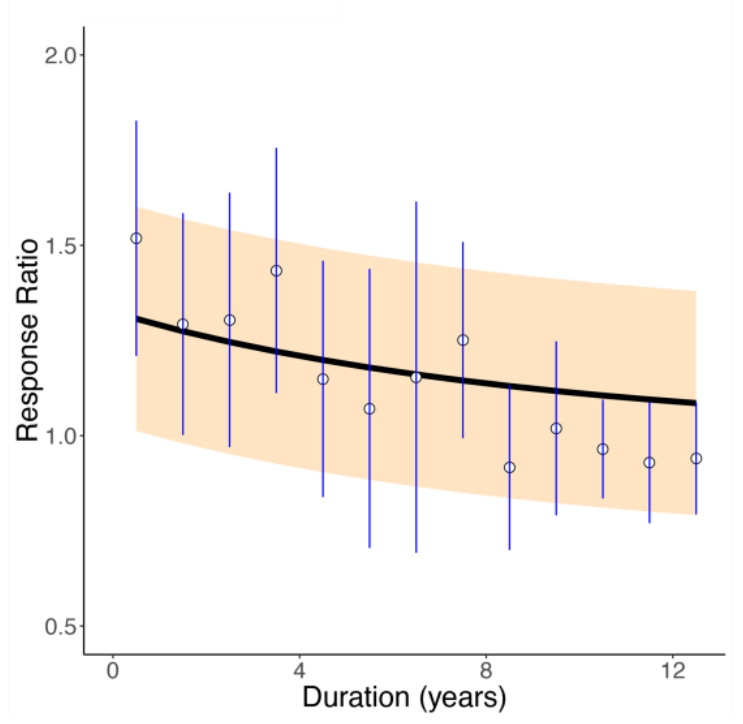

(d)

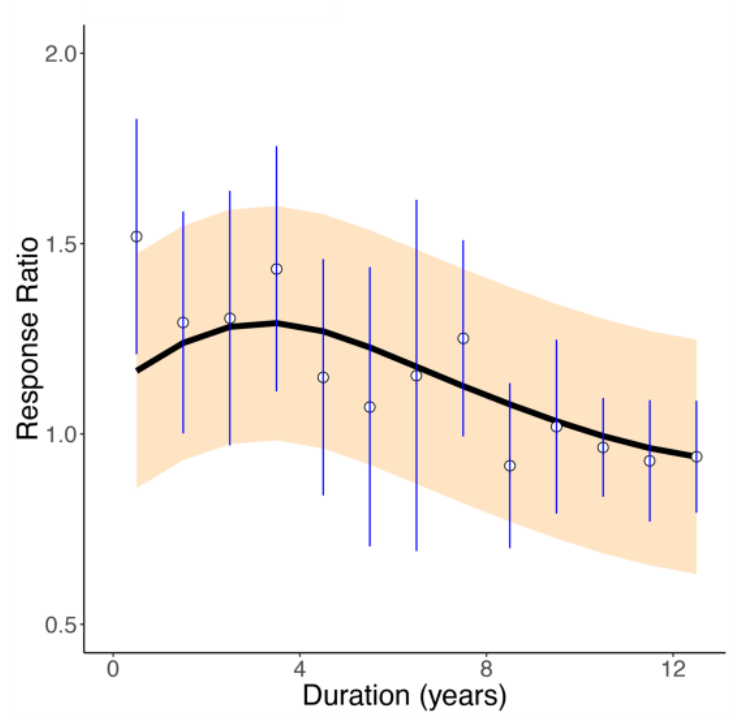


224 Figure S2: 95\% probability density credible areas for (a) AWB $V_{U_{r e f}}$; and (b) $m_{t}$ parameters corresponding to prewarming steady state $\mathrm{SOC}=100 \mathrm{mg} \mathrm{C} \mathrm{g}^{-1}$ soil, $\mathrm{DOC}=0.2 \mathrm{mg} \mathrm{C} \mathrm{g}^{-1}$ soil, $\mathrm{MIC}=2 \mathrm{mg} \mathrm{C} \mathrm{g}^{-1}$ soil, and $\mathrm{ENZ}=0.1 \mathrm{mg}$ $226 \mathrm{C} \mathrm{g}^{-1}$ soil. Yellow shaded regions represent $80 \%$ credible areas and vertical purple lines indicate distribution mean.

(a)

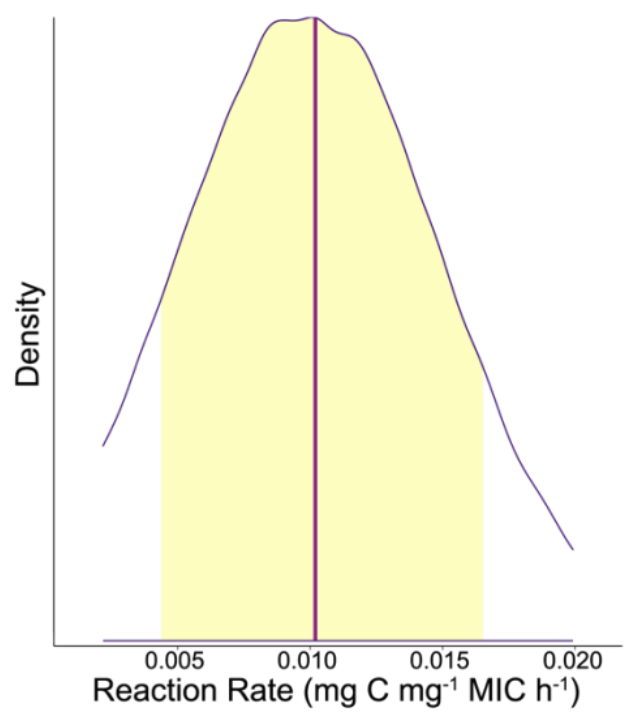

(b)

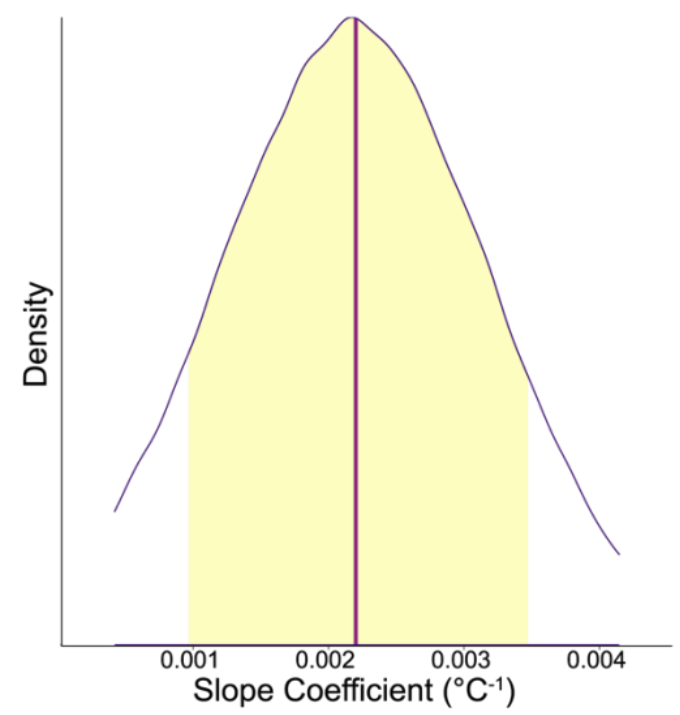


258 Figure S3: Response ratios of model SOC stocks after 12.5 years of warming in AWB and CON simulations. (a) Pre-warming steady state SOC varied from 50 to $200 \mathrm{mg} \mathrm{C} \mathrm{g}^{-1}$ soil, with pre-warming MIC, DOC and ENZ held

260 constant respectively at $2 \mathrm{mg} \mathrm{C} \mathrm{g}^{-1}$ soil, $0.2 \mathrm{mg} \mathrm{C} \mathrm{g}^{-1}$ soil, and $0.1 \mathrm{mg} \mathrm{C} \mathrm{g}^{-1}$ soil; (b), Pre-warming MIC varied from 1 to $8 \mathrm{mg} \mathrm{C} \mathrm{g}^{-1}$ soil, with pre-warming SOC, DOC and ENZ held constant, respectively, at $100 \mathrm{mg} \mathrm{C} \mathrm{g}^{-1}$ soil, $0.2 \mathrm{mg}$ $262 \mathrm{C} \mathrm{g}^{-1}$ soil, and $0.1 \mathrm{mg} \mathrm{C} \mathrm{g}^{-1}$ soil.

(a)

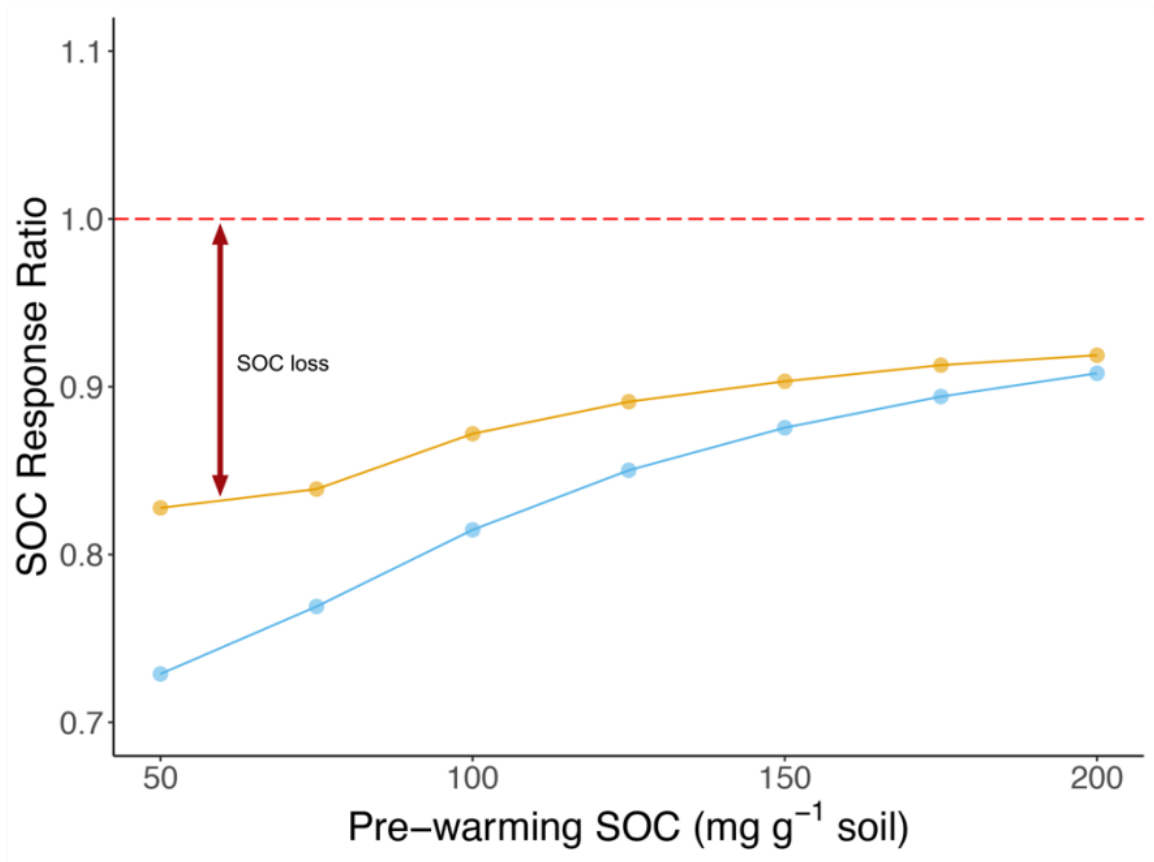

(b)

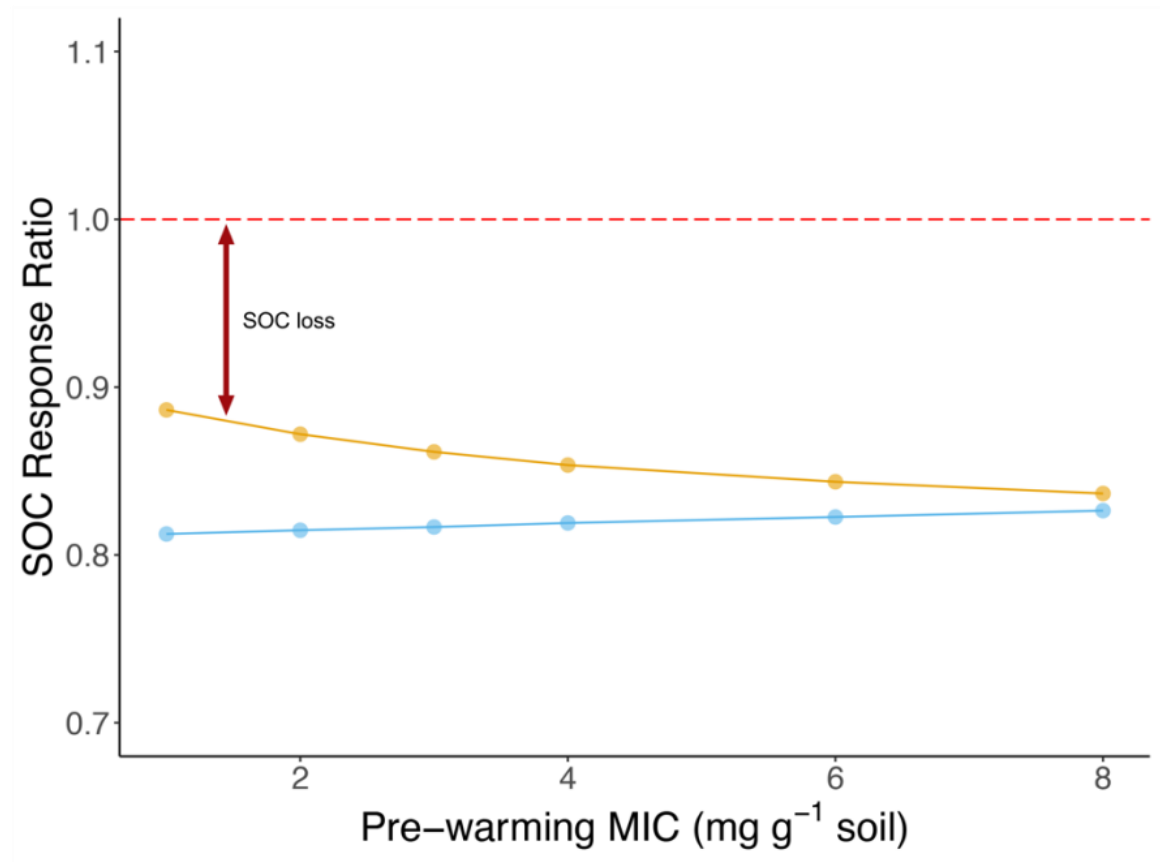


264 Table S2: (a) AWB; and (b) CON prior distribution tables. Including $\sigma$, the residual error scale term, we fit 10 parameters in our AWB runs and 8 parameters in our CON runs. Normal, Gaussian priors were used for all fitted

266 ODE model parameters. The notation we use for our normal distributions follows an N(mean, standard deviation) format. The Markov chain guess-scaling parameter, $\sigma$, was drawn from a more weakly informative half-Cauchy

268 distribution per recommendations from literature (Gelman, 2006).

$270 \quad$ (a) $\mathrm{CON}$ priors

$\begin{array}{lll}\text { Parameter } & \text { Distribution } & \text { Parameter Description } \\ E a_{S} & \mathrm{~N}(50,25) & \text { SOC activation energy } \\ E a_{D} & \mathrm{~N}(50,25) & \text { DOC activation energy } \\ E a_{M} & \mathrm{~N}(50,25) & \text { MIC activation energy } \\ a_{D S} & \mathrm{~N}(0.3,0.15) & \text { DOC to SOC transfer coefficient } \\ a_{S D} & \mathrm{~N}(0.3,0.15) & \text { SOC to DOC transfer coefficient } \\ a_{M} & \mathrm{~N}(0.3,0.15) & \text { MIC to SOC transfer coefficient } \\ a_{M S} & \mathrm{~N}(0.5,0.25) & \text { Fraction of dead MIC transferred } \\ \sigma & \text { Cauchy }(0,1) & \text { Residual Error Scale }\end{array}$

272 (b) AWB priors

$\begin{array}{lll}\text { Parameter } & \text { Distribution } & \text { Parameter Description } \\ V_{\text {ref }} & \mathrm{N}(0.4,0.2) & \text { SOC reference } V_{\text {max }} \\ V_{U_{r e f}} & \mathrm{~N}(0.01,0.005) & \text { DOC reference } V_{\text {max }} \\ E a_{V} & \mathrm{~N}(50,25) & \text { SOC } V_{\text {max }} \text { activation energy } \\ E a_{V U} & \mathrm{~N}(50,25) & \text { DOC } V_{\text {max }} \text { activation energy } \\ E a_{K} & \mathrm{~N}(50,25) & \text { SOC } K_{M} \text { activation energy } \\ E a_{K U} & \mathrm{~N}(50,25) & \text { DOC } K_{M} \text { activation energy } \\ E_{C_{r e f}} & \mathrm{~N}(0.4,0.2) & \text { Reference C use efficiency (CUE) } \\ m_{t} & \mathrm{~N}(0.002,0.001) & \text { CUE slope } \\ a_{M S} & \mathrm{~N}(0.5,0.25) & \text { Fraction of dead MBC transferred to SOC } \\ \sigma & \text { Cauchy }(0,1) & \text { Residual Error Scale }\end{array}$


Table S3: Posterior means calculated for parameters that were fit in $\mathrm{HMC}$ runs are displayed in the following tables. Tables are presented in the order of (a) CON SOC-varied runs; (b) AWB SOC-varied runs; (c) CON MICvaried runs; and d) AWB MIC-varied runs.

294

(a) $\mathrm{CON}$ posterior distribution means for SOC-varied runs

$\begin{array}{lllllllll}\text { Parameter } & \text { SOC50 } & \text { SOC75 } & \text { SOC100 } & \text { SOC125 } & \text { SOC150 } & \text { SOC175 } & \text { SOC200 } \\ E a_{S} & 77.565 & 73.582 & 66.578 & 60.796 & 56.695 & 53.602 & 51.347 \\ E a_{D} & 50.24 & 50.17 & 50.133 & 50.211 & 50.174 & 50.25 & 50.246 \\ E a_{M} & 52.612 & 52.531 & 52.143 & 52.109 & 52.07 & 51.989 & 51.836 \\ a_{D S} & 0.324 & 0.325 & 0.327 & 0.327 & 0.327 & 0.327 & 0.326 \\ a_{S D} & 0.334 & 0.336 & 0.336 & 0.337 & 0.337 & 0.337 & 0.337 \\ a_{M} & 0.338 & 0.34 & 0.336 & 0.334 & 0.333 & 0.332 & 0.331 \\ a_{M S} & 0.504 & 0.504 & 0.502 & 0.5 & 0.498 & 0.497 & 0.497 \\ \sigma & 0.134 & 0.139 & 0.155 & 0.168 & 0.176 & 0.183 & 0.187\end{array}$

298 (b) AWB posterior distribution means for SOC-varied runs

$\begin{array}{llllllll}\text { Parameter } & \text { SOC50 } & \text { SOC75 } & \text { SOC100 } & \text { SOC125 } & \text { SOC150 } & \text { SOC175 } & \text { SOC200 } \\ V_{\text {ref }} & 0.383 & 0.405 & 0.41 & 0.414 & 0.418 & 0.42 & 0.423 \\ V_{U_{r e f}} & 0.0104 & 0.0104 & 0.0104 & 0.0104 & 0.0104 & 0.0104 & 0.0104 \\ E a_{V} & 74.791 & 70.071 & 65.21 & 62.218 & 60.447 & 59.174 & 58.475 \\ E a_{V U} & 50.201 & 50.486 & 50.669 & 50.823 & 51.104 & 51.015 & 51.106 \\ E a_{K} & 25.846 & 30.357 & 35.532 & 38.395 & 40.214 & 41.404 & 42.364 \\ E a_{K U} & 49.791 & 49.671 & 49.318 & 49.265 & 49.014 & 49.013 & 48.964 \\ E_{C_{r e f}} & 0.204 & 0.253 & 0.337 & 0.406 & 0.454 & 0.49 & 0.513 \\ m_{t} & 0.00184 & 0.00214 & 0.00222 & 0.00225 & 0.00227 & 0.00229 & 0.00232 \\ a_{M S} & 0.495 & 0.498 & 0.5 & 0.507 & 0.514 & 0.52 & 0.526 \\ \sigma & 0.152 & 0.151 & 0.16 & 0.165 & 0.17 & 0.174 & 0.178\end{array}$

300 (c) CON posterior distribution means for MIC-varied runs

Parameter MIC1 MIC2 MIC3 MIC4 MIC6 MIC8

$\begin{array}{lllllll}E a_{S} & 67.472 & 66.578 & 65.991 & 65.34 & 64.076 & 62.972\end{array}$

$\begin{array}{lllllll}E a_{D} & 49.941 & 50.133 & 50.337 & 50.388 & 50.608 & 50.801\end{array}$

$\begin{array}{llllllll}E a_{M} & 50.909 & 52.143 & 53.063 & 53.862 & 54.861 & 55.681\end{array}$

$\begin{array}{lllllll}\mathrm{a}_{\mathrm{DS}} & 0.327 & 0.327 & 0.326 & 0.327 & 0.327 & 0.327\end{array}$

$\begin{array}{lllllll}a_{S D} & 0.332 & 0.336 & 0.339 & 0.342 & 0.344 & 0.346\end{array}$

$\begin{array}{llllllll}a_{M} & 0.336 & 0.336 & 0.336 & 0.336 & 0.336 & 0.335\end{array}$

$\begin{array}{llllllll}a_{M S} & 0.503 & 0.502 & 0.499 & 0.498 & 0.496 & 0.495\end{array}$

$\begin{array}{lllllll}\sigma & 0.155 & 0.155 & 0.155 & 0.155 & 0.156 & 0.156\end{array}$

(d) AWB posterior distribution means for MIC-varied runs

Parameter MIC1 MIC2 MIC3 MIC4 MIC6 MIC8

$\begin{array}{llllllll}V_{\text {ref }} & 0.403 & 0.41 & 0.413 & 0.417 & 0.422 & 0.424\end{array}$




$\begin{array}{lllllll}V_{U_{\text {ref }}} & 0.0105 & 0.0104 & 0.0104 & 0.0104 & 0.0104 & 0.0104 \\ E a_{V} & 65.571 & 65.21 & 65.207 & 65.308 & 65.689 & 66.111 \\ E a_{V U} & 50.719 & 50.669 & 50.67 & 50.601 & 50.56 & 50.461 \\ E a_{K} & 34.939 & 35.532 & 35.585 & 35.467 & 35.071 & 34.936 \\ E a_{K U} & 49.441 & 49.318 & 49.4 & 49.403 & 49.608 & 49.495 \\ E_{C_{r e f}} & 0.26 & 0.337 & 0.395 & 0.437 & 0.495 & 0.5343 \\ m_{t} & 0.00218 & 0.00222 & 0.00223 & 0.00224 & 0.00226 & 0.00227 \\ a_{M S} & 0.487 & 0.5 & 0.516 & 0.534 & 0.564 & 0.581 \\ \sigma & 0.16 & 0.16 & 0.16 & 0.161 & 0.163 & 0.164\end{array}$

304

306

308

310

312

314

316

318

320

322

324

326

328

330

332

334

336

338

340

342

344

346 
Table S4: Numerical LOO, WAIC, LPML, and $\mathrm{R}^{2}$ results are displayed in the following tables. Lower LOO and WAIC values are preferred. Higher LPML values are preferred. Tables are presented in the order of (a) CON SOCvaried runs; (b) AWB SOC-varied runs; (c) CON MIC-varied runs; and d) AWB MIC-varied runs.

(a) CON goodness-of-fit metrics for SOC-varied runs

Parameter SOC50 SOC75 SOC100 SOC125 SOC150 SOC175 SOC200

$\begin{array}{llllllll}\text { LOO } & -15.704 & -14.929 & -11.918 & -9.844 & -8.51 & -7.574 & -6.891 \\ \text { WAIC } & -15.818 & -15.002 & -11.992 & -9.92 & -8.58 & -7.639 & -6.966 \\ \text { LPML } & 7.849 & 7.465 & 5.959 & 4.92 & 4.256 & 3.788 & 3.439 \\ \text { R }^{2} & 0.627 & 0.596 & 0.496 & 0.413 & 0.351 & 0.304 & 0.269\end{array}$

(b) AWB goodness-of-fit metrics for SOC-varied runs

Parameter SOC50 SOC75 SOC100 SOC125 SOC150 SOC175 SOC200

$\begin{array}{llllllll}\text { LOO } & -11.028 & -10.633 & -8.388 & -7.732 & -7.084 & -6.525 & -5.97 \\ \text { WAIC } & -11.379 & -11.123 & -9.284 & -8.446 & -7.733 & -7.114 & -6.579 \\ \text { LPML } & 5.499 & 5.312 & 4.252 & 3.9 & 3.547 & 3.254 & 3.002 \\ \text { R }^{2} & 0.572 & 0.585 & 0.528 & 0.492 & 0.463 & 0.435 & 0.406\end{array}$

(c) CON goodness-of-fit metrics for MIC-varied runs

Parameter MIC1 MIC2 MIC3 MIC4 MIC6 MIC8

$\begin{array}{lllllll}\text { LOO } & -11.963 & -11.918 & -11.931 & -11.887 & -11.808 & -11.731\end{array}$

$\begin{array}{lllllll}\text { WAIC } & -12.035 & -11.992 & -12.004 & -11.966 & -11.881 & -11.802\end{array}$

$\begin{array}{lllllll}\text { LPML } & 5.982 & 5.959 & 5.966 & 5.943 & 5.904 & 5.865\end{array}$

$\begin{array}{llllllll}\mathrm{R}^{2} & 0.498 & 0.496 & 0.496 & 0.496 & 0.493 & 0.49\end{array}$

356

(d) AWB goodness-of-fit metrics for MIC-varied runs

Parameter MIC1 MIC2 MIC3 MIC4 MIC6 MIC8

$\begin{array}{llllllll}\text { LOO } & -8.63 & -8.388 & -8.587 & -8.462 & -8.219 & -8.181\end{array}$

$\begin{array}{lllllll}\text { WAIC } & -9.302 & -9.284 & -9.213 & -9.079 & -8.863 & -8.711\end{array}$

$\begin{array}{lllllll}\text { LPML } & 4.314 & 4.252 & 4.204 & 4.203 & 4.116 & 4.088\end{array}$

$\begin{array}{lllllll}\mathrm{R}^{2} & 0.526 & 0.528 & 0.525 & 0.521 & 0.516 & 0.513\end{array}$ 
Figure S4: Change in fit metrics for $\mathrm{AWB}$ and CON as pre-warming steady state MIC is varied from 1 to $8 \mathrm{mg} \mathrm{C}^{-1}$ soil. (a) LOO; (b) WAIC; (c) LPML; (d) $\mathrm{R}^{2}$
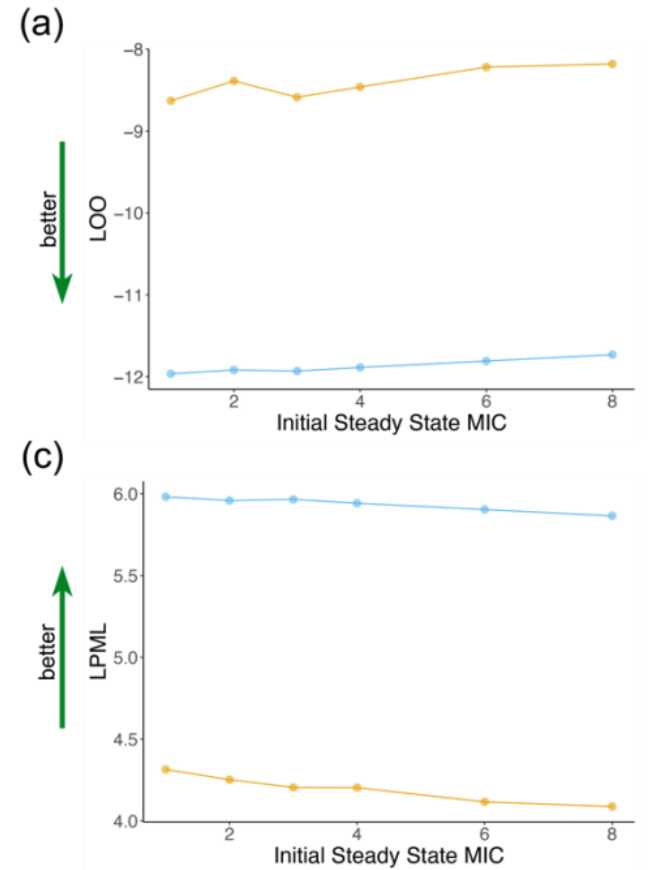
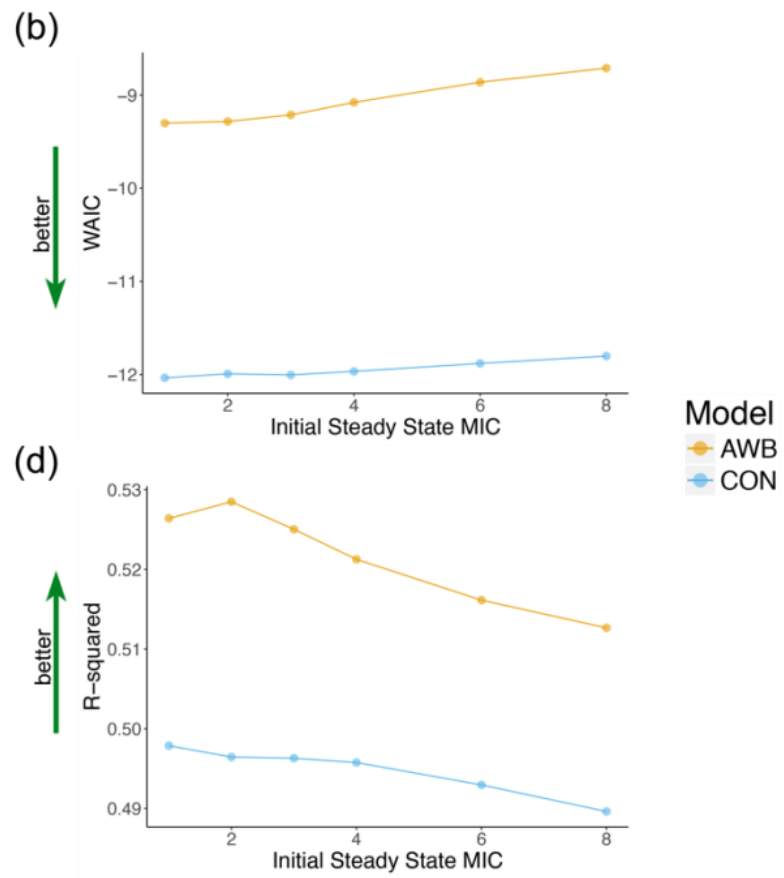
Figure S5: Trace plots for AWB and CON parameters indicate that the Markov chains were well-mixed with appropriate burn-in. Example trace plots depicted in which pre-warming $\mathrm{SOC}=100 \mathrm{mg} \mathrm{C} \mathrm{g}^{-1}$ soil, $\mathrm{MIC}=2 \mathrm{mg} \mathrm{C} \mathrm{g}^{-}$ ${ }^{1}$ soil, $\mathrm{DOC}=0.2 \mathrm{mg} \mathrm{C} \mathrm{g}^{-1}$ soil, and (for $\mathrm{AWB}$ ) $\mathrm{ENZ}=0.1 \mathrm{mg} \mathrm{C} \mathrm{g}^{-1}$ soil. (a) CON $E a$ parameters; (b) CON partition fraction parameters; (c) AWB Ea parameters; (d) AWB parameters $V_{r e f}, E_{C_{r e f}}, a_{M S}, V_{U_{r e f}}$, and $m_{t}$. The red ticks at the bottom of the AWB panels indicate divergent transitions on one out of the four chains.

(a)
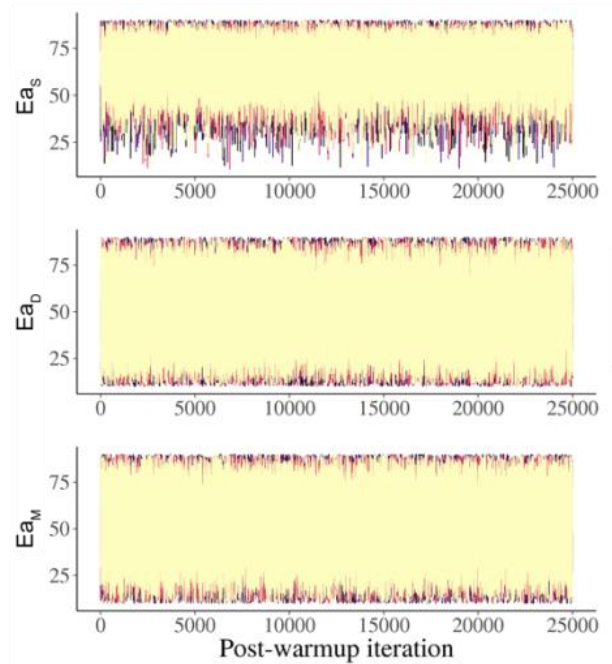

(c)
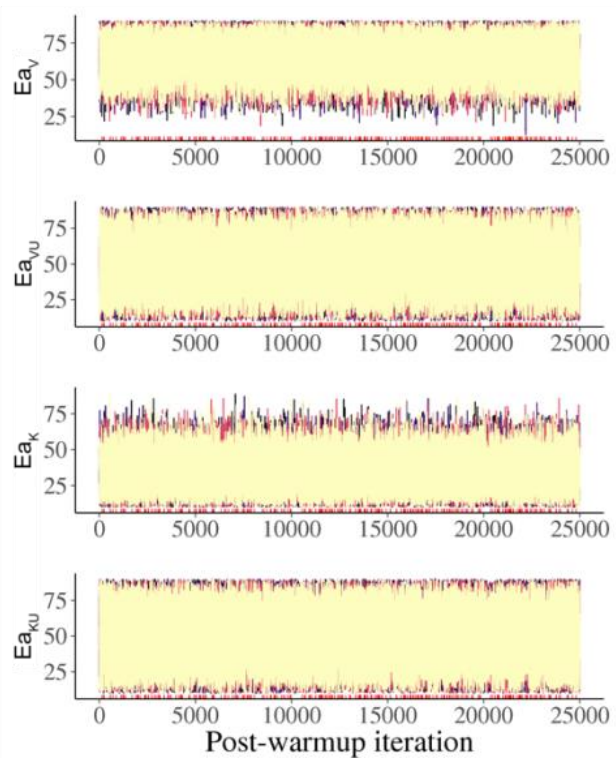

(b)
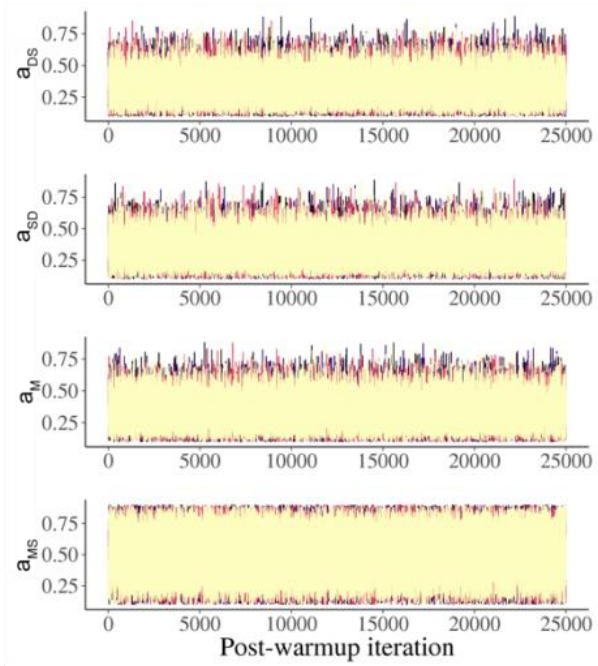

(d)
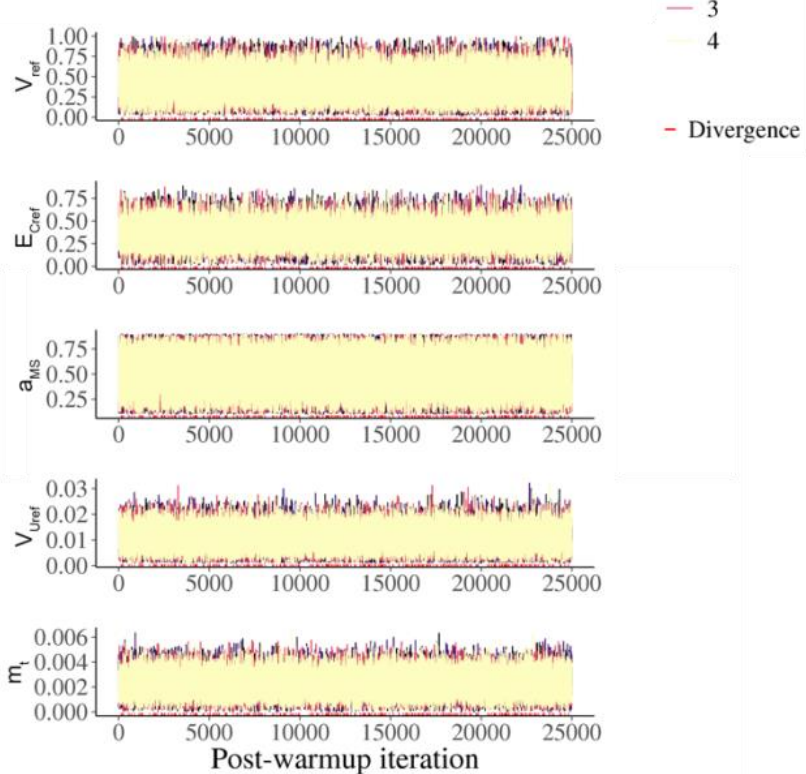
424 Figure S6: Autocorrelation plots for pre-warming SOC $=100 \mathrm{mg} \mathrm{C} \mathrm{g}^{-1}$ soil, $\mathrm{MIC}=2 \mathrm{mg} \mathrm{C} \mathrm{g}^{-1}$ soil, DOC $=0.2 \mathrm{mg}$ $\mathrm{C} \mathrm{g}^{-1}$ soil, and (for $\mathrm{AWB}$ ) $\mathrm{ENZ}=0.1 \mathrm{mg} \mathrm{C} \mathrm{g}^{-1}$ soil indicate effective sample collection. For all fitted AWB and CON parameters, autocorrelation, or the dependence between values of the same parameter accepted by Markov chains, tends to drop as lag, the distance between MCMC iterations increases. Low autocorrelation indicates more independence between samples and more efficient collection of effective samples for inference. (a) CON Ea parameters; (b) CON partition fraction parameters; (c) AWB Ea parameters; (d) AWB parameters $V_{r e f}, E_{C_{r e f}}, a_{M S}$, $V_{U_{r e f}}$, and $m_{t}$.

(a)

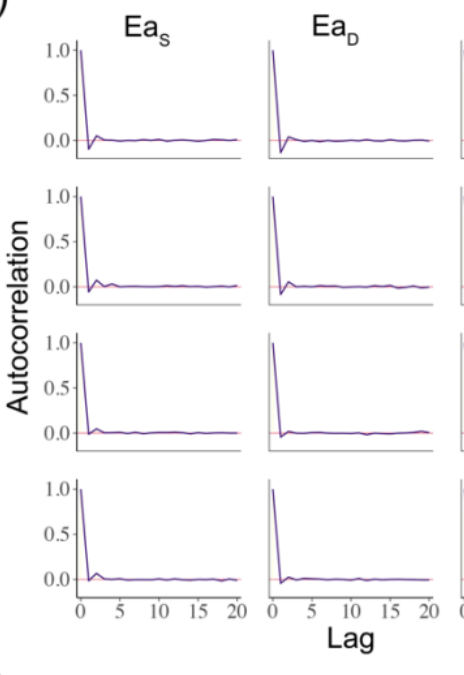

(c)
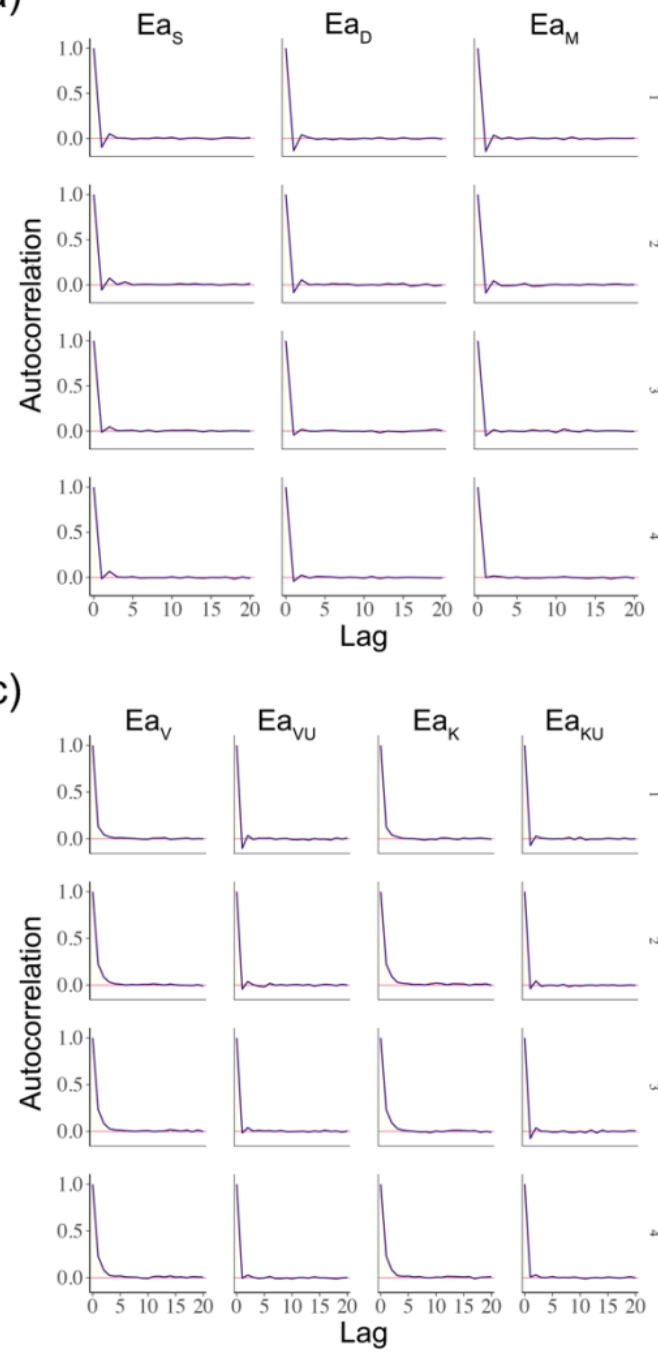

(b)

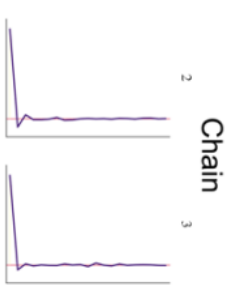

(d)
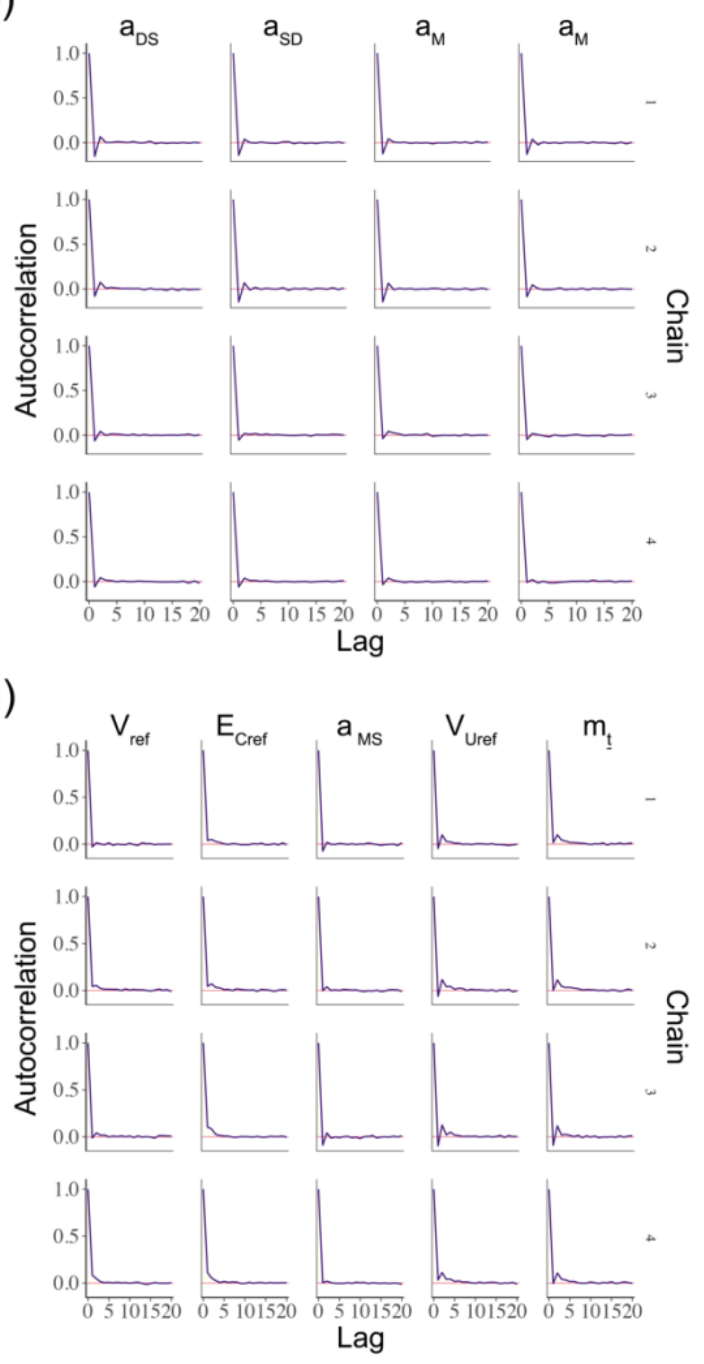
440 Figure S7: $\hat{R}$ is a Bayesian diagnostic measure that estimates the degree of convergence between multiple Markov chains. An $\hat{R}$ value that approaches 1 as the number of Markov chain iterations increase is ideal. Plots demonstrating 442 convergence of $\hat{R}$ values to 1 are presented for (a) CON; and (b) AWB parameters corresponding to simulations using pre-warming $\mathrm{SOC}=100 \mathrm{mg} \mathrm{C} \mathrm{g}^{-1}$ soil, MIC $=2 \mathrm{mg} \mathrm{C} \mathrm{g}^{-1}$ soil, DOC $=0.2 \mathrm{mg} \mathrm{C} \mathrm{g}^{-1}$ soil, and (for AWB) ENZ $444=0.1 \mathrm{mg} \mathrm{C} \mathrm{g}^{-1}$ soil.

(a)
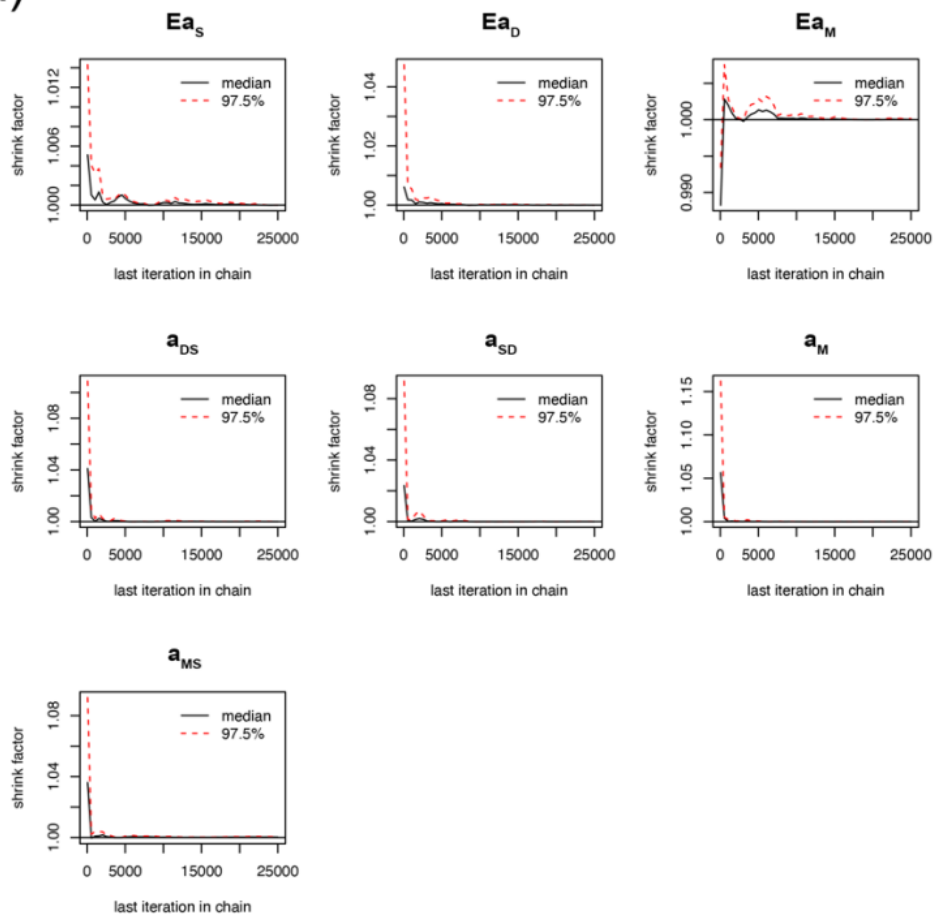

(b)
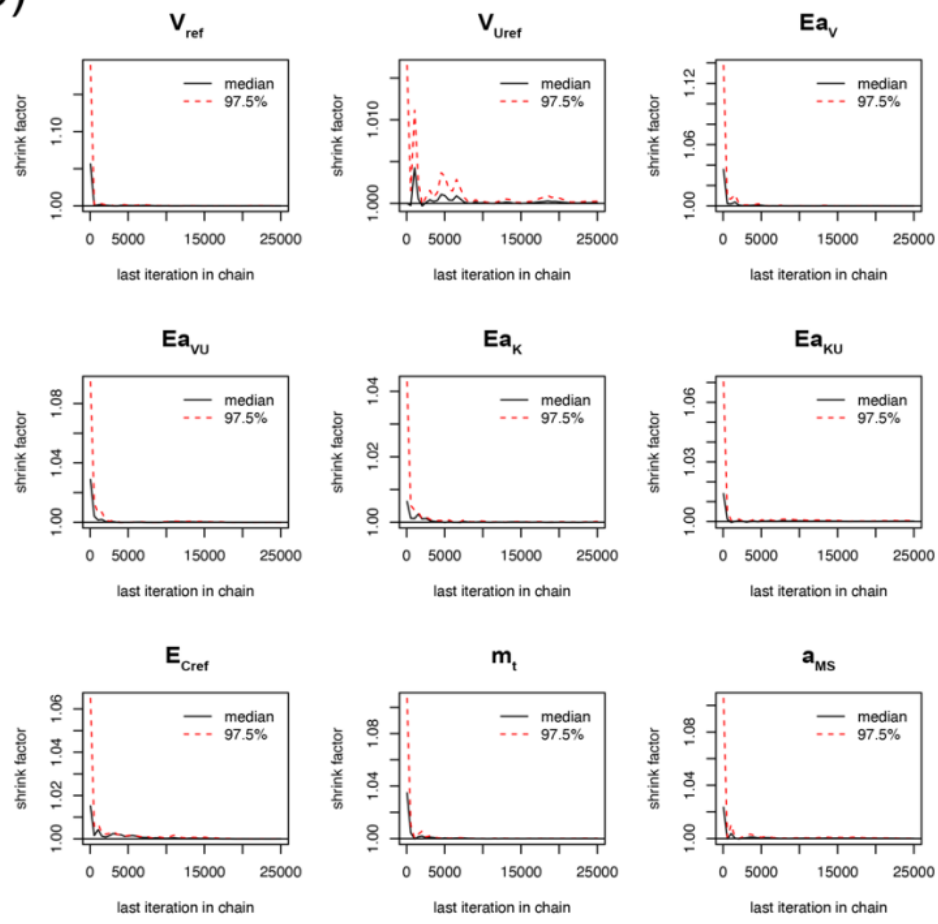
Table S5: CON and AWB model parameter effective sample size fractions $\mathrm{N}_{\text {eff }} / \mathrm{N}$ (ratio of effective posterior samples to total posterior samples where $\mathrm{N}=100,000)$ calculations from posterior. Ratios that are closer to 1.0 are preferred. Tables are presented in the order of (a) CON SOC-varied runs; (b) AWB SOC-varied runs; (c) CON MIC-varied runs; and d) AWB MIC-varied runs.

(a) CON SOC-varied runs

Parameter SOC50 SOC75 SOC100 SOC125 SOC150 SOC175 SOC200

$\begin{array}{llllllll}E a_{S} & 0.877 & 0.848 & 0.935 & 0.924 & 0.867 & 0.884 & 0.87 \\ E a_{D} & 1.0 & 1.0 & 1.0 & 1.0 & 1.0 & 1.0 & 1.0 \\ E a_{M} & 1.0 & 1.0 & 1.0 & 1.0 & 1.0 & 1.0 & 1.0 \\ a_{D S} & 0.974 & 0.949 & 0.974 & 1.0 & 1.0 & 0.953 & 1.0 \\ a_{S D} & 0.95 & 0.957 & 1.0 & 1.0 & 0.925 & 1.0 & 1.0 \\ a_{M} & 0.93 & 0.919 & 1.0 & 1.0 & 0.987 & 0.987 & 0.955 \\ a_{M S} & 1.0 & 1.0 & 1.0 & 1.0 & 1.0 & 1.0 & 1.0 \\ \sigma & 0.686 & 0.653 & 0.719 & 0.732 & 0.693 & 0.689 & 0.691\end{array}$

(b) AWB SOC-varied runs

Parameter SOC50 SOC75 SOC100 SOC125 SOC150 SOC175 SOC200

$\begin{array}{llllllll}V_{\text {ref }} & 0.594 & 0.883 & 0.81 & 0.914 & 0.959 & 0.816 & 0.707\end{array}$

$\begin{array}{llllllll}V_{U_{\text {ref }}} & 0.699 & 0.705 & 0.704 & 0.723 & 0.694 & 0.64 & 0.629\end{array}$

$\begin{array}{llllllll}E a_{V} & 0.568 & 0.687 & 0.579 & 0.561 & 0.511 & 0.413 & 0.423\end{array}$

$\begin{array}{llllllll}E a_{V U} & 0.961 & 1.0 & 1.0 & 1.0 & 1.0 & 0.95 & 0.845\end{array}$

$\begin{array}{llllllll}E a_{K} & 0.463 & 0.687 & 0.599 & 0.527 & 0.519 & 0.424 & 0.428\end{array}$

$\begin{array}{lllllllll}E a_{K U} & 0.995 & 1.0 & 1.0 & 1.0 & 1.0 & 0.949 & 0.828\end{array}$

$\begin{array}{llllllll}E_{C_{\text {ref }}} & 0.439 & 0.635 & 0.698 & 0.65 & 0.458 & 0.339 & 0.261\end{array}$

$\begin{array}{llllllll}m_{t} & 0.625 & 0.668 & 0.679 & 0.722 & 0.625 & 0.638 & 0.556\end{array}$

$\begin{array}{llllllll}a_{M S} & 1.0 & 1.0 & 1.0 & 1.0 & 1.0 & 0.879 & 0.793\end{array}$

$\begin{array}{llllllll}\sigma & 0.529 & 0.638 & 0.612 & 0.65 & 0.681 & 0.579 & 0.533\end{array}$

454

(c) CON MIC-varied runs

Parameter MIC1 MIC2 MIC3 MIC4 MIC6 MIC8

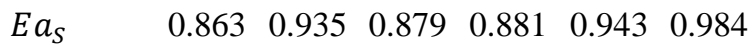

$\begin{array}{lllllll}E a_{D} & 1.0 & 1.0 & 1.0 & 1.0 & 1.0 & 1.0\end{array}$

$\begin{array}{lllllll}E a_{M} & 1.0 & 1.0 & 1.0 & 1.0 & 1.0 & 1.0\end{array}$

$\begin{array}{lllllll}a_{D S} & 0.968 & 0.974 & 0.964 & 0.966 & 0.936 & 1.0\end{array}$

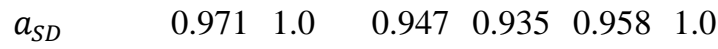

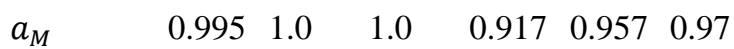

$\begin{array}{lllllll}a_{M S} & 1.0 & 1.0 & 1.0 & 1.0 & 1.0 & 1.0\end{array}$

$\begin{array}{llllllll}\sigma & 0.68 & 0.719 & 0.699 & 0.659 & 0.689 & 0.747\end{array}$

(d) AWB MIC-varied runs

Parameter MIC1 MIC2 MIC3 MIC4 MIC6 MIC8

$\begin{array}{llllllll}V_{\text {ref }} & & 0.673 & 0.81 & 0.84 & 0.761 & 0.851 & 0.907\end{array}$ 


$\begin{array}{llllllll} & V_{U_{\text {ref }}} & 0.705 & 0.704 & 0.693 & 0.62 & 0.722 & 0.702 \\ E a_{V} & 0.536 & 0.579 & 0.611 & 0.563 & 0.621 & 0.632 \\ & E a_{V U} & 0.968 & 1.0 & 1.0 & 0.935 & 1.0 & 1.0 \\ E a_{K} & 0.44 & 0.599 & 0.403 & 0.523 & 0.622 & 0.609 \\ E a_{K U} & 0.985 & 1.0 & 1.0 & 0.921 & 1.0 & 1.0 \\ & E_{C r e f} & 0.486 & 0.698 & 0.432 & 0.582 & 0.534 & 0.547 \\ & m_{t} & 0.539 & 0.679 & 0.739 & 0.632 & 0.641 & 0.729 \\ & a_{M S} & 0.973 & 1.0 & 1.0 & 0.898 & 0.895 & 0.954 \\ 458 & \sigma & 0.564 & 0.612 & 0.697 & 0.548 & 0.579 & 0.621\end{array}$

460

462

464

466

468

470

472

474

476

478

480

482

484

486

488

490

492 
Table S6: Pareto $k$ diagnostic counts for CON and AWB simulations sourced from LOO metric computations for evaluate LOO to the shape parameter of a Pareto distribution. In our case, each simulation will produce $13 k$ CON and AWB model fits. $k$ diagnostics are calculated by fitting each set of leave-one-out importance ratios used to parameters since there are 13 meta-analysis data points and consequently the same number of holdout sets. $k$ gives an indication on model quality and LOO result reliability. Having a higher proportion of lower $k$ values are preferred; samples are suitable if they fall within the intervals of $(-\infty, 0.5)$ and $(0.5,0.7)$ and questionable if they are higher. Having a high proportion of $k$ values greater than 1 should raise concerns of unreliable information criteria and cross validation results stemming from model specification issues. The presence of a diagnostic for LOO renders it a superior Bayesian predictive metric; other predictive metrics lack reviewable diagnostics. Tables are presented in the order of (a) CON SOC-varied runs; (b) AWB SOC-varied runs; (c) CON MIC-varied runs; and d)

504 AWB MIC-varied runs.

(a) CON SOC-varied runs

$k$ interval SOC50 SOC75 SOC100 SOC125 SOC150 SOC175 SOC200

506

$\begin{array}{llllllll}(-\infty, 0.5] & 13 & 13 & 13 & 13 & 13 & 13 & 12 \\ (0.5,0.7] & 0 & 0 & 0 & 0 & 0 & 0 & 1 \\ (0.7,1] & 0 & 0 & 0 & 0 & 0 & 0 & 0 \\ (1, \infty] & 0 & 0 & 0 & 0 & 0 & 0 & 0\end{array}$

(b) AWB SOC-varied runs

$k$ interval SOC50 SOC75 SOC100 SOC125 SOC150 SOC175 SOC200

508

$\begin{array}{llllllll}(-\infty, 0.5] & 11 & 11 & 12 & 12 & 12 & 11 & 12 \\ (0.5,0.7] & 2 & 2 & 0 & 0 & 0 & 2 & 1 \\ (0.7,1] & 0 & 0 & 1 & 1 & 1 & 0 & 0 \\ (1, \infty] & 0 & 0 & 0 & 0 & 0 & 0 & 0\end{array}$

(c) CON MIC-varied runs

$\begin{array}{lllllll}k \text { interval } & \text { MIC1 } & \text { MIC2 } & \text { MIC3 } & \text { MIC4 } & \text { MIC6 } & \text { MIC8 } \\ (-\infty, 0.5] & 13 & 13 & 13 & 13 & 13 & 13 \\ (0.5,0.7] & 0 & 0 & 0 & 0 & 0 & 0 \\ (0.7,1] & 0 & 0 & 0 & 0 & 0 & 0 \\ (1, \infty] & 0 & 0 & 0 & 0 & 0 & 0\end{array}$

510

(d) AWB MIC-varied runs

$\begin{array}{lllllll}k \text { interval } & \text { MIC1 } & \text { MIC2 } & \text { MIC3 } & \text { MIC4 } & \text { MIC6 } & \text { MIC8 } \\ (-\infty, 0.5] & 12 & 12 & 12 & 11 & 12 & 12 \\ (0.5,0.7] & 0 & 0 & 1 & 2 & 0 & 1 \\ (0.7,1] & 1 & 1 & 0 & 0 & 1 & 0 \\ (1, \infty] & 0 & 0 & 0 & 0 & 0 & 0\end{array}$


Figure S8: Plots of effective parameter counts for CON and AWB in SOC-varied and MIC-varied HMC runs. Decreasing SOC in AWB and CON runs increased effective parameter count and over-fitting punishment in the LOO and WAIC calculations. Effective parameter counts computed as part of (a) LOO for SOC-varied runs; (b) 524 WAIC for SOC-varied runs; (c) LOO for MIC-varied runs; and (d) WAIC for MIC-varied runs.

(a)

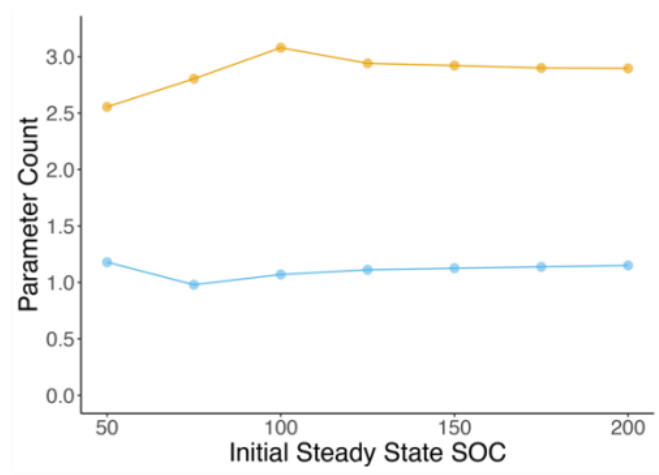

(c)

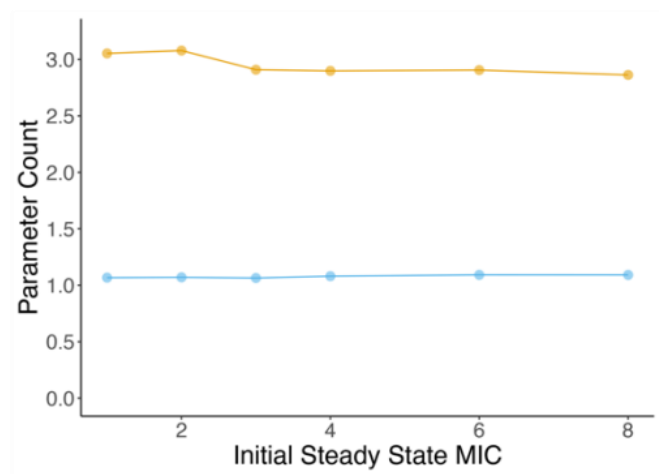

(b)

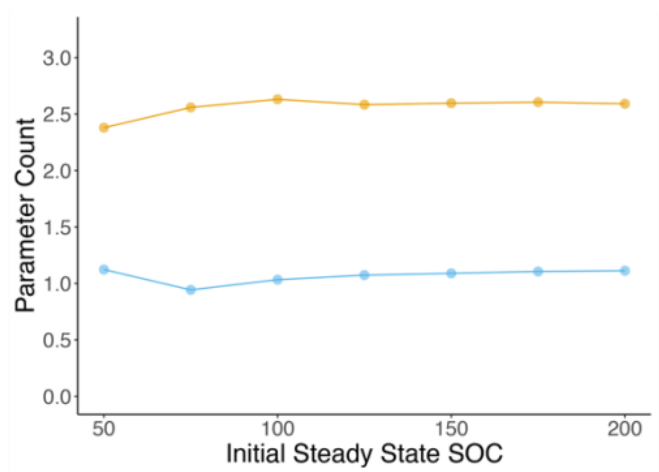

(d)

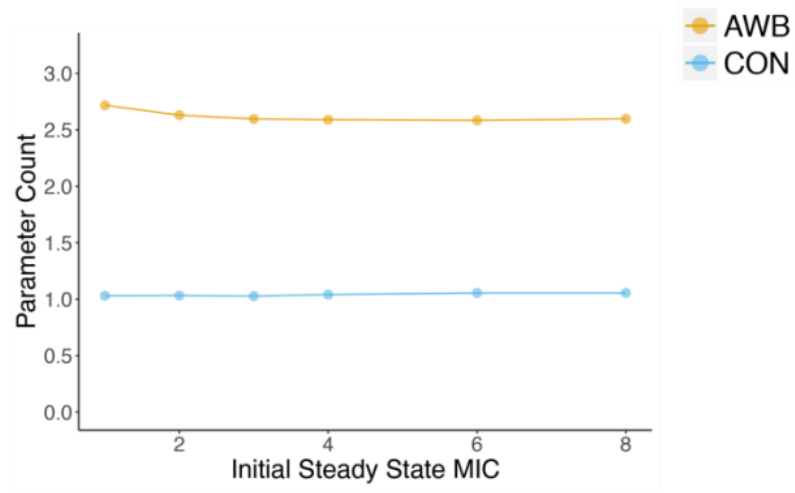


Figure S9: Ratio of divergent transitions to total posterior samples collected in AWB runs. Decreasing the MIC-toSOC ratio in AWB runs corresponded to an increase in the number of divergent transitions. Divergent transition frequencies per 100,000 posterior samples in (a) SOC-varied runs; and (b) in MIC-varied runs.

(a)

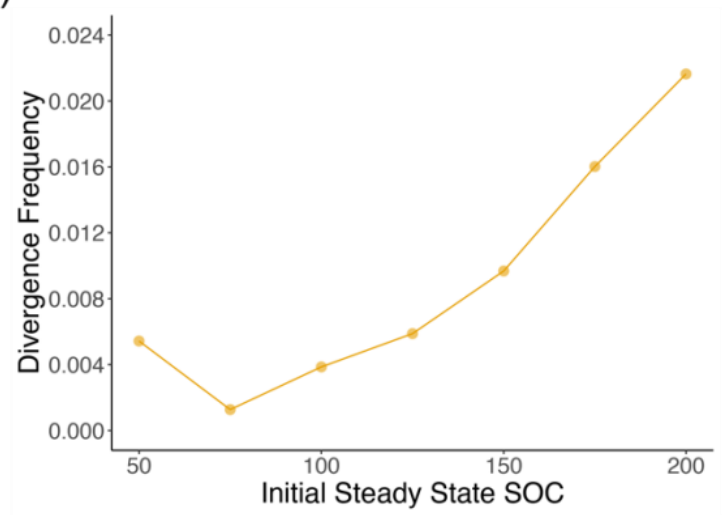

(b)

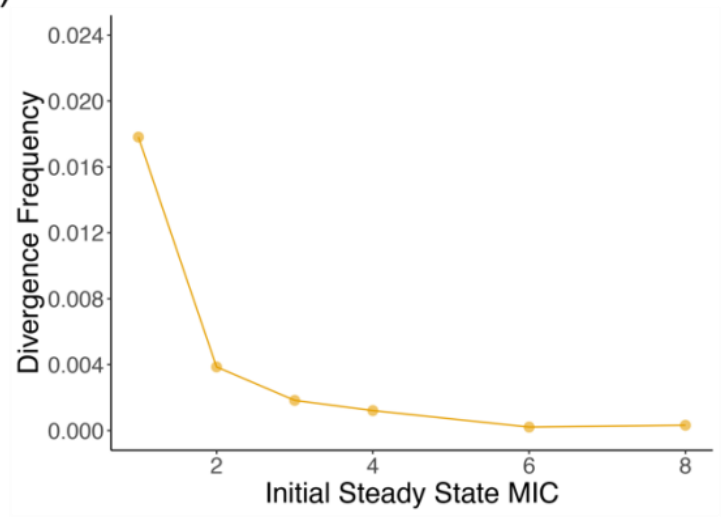


590 Figure S10: Response ratios of empirical SOC stocks from 143 field warming studies (van Gestel et al., 2018) plotted against study duration. A statistical analysis not accounting for sample size of each study found that the

592 effect of study duration on response ratio was not significant $(\mathrm{p}=0.7822)$. Response ratios ranged from 0.544 to 1.9. Mean response ratio was 1.03 , not accounting for sample sizes. The red dashed line at 1.0 divides studies in which

594 an increase in SOC was observed from those in which a decrease was ultimately observed.

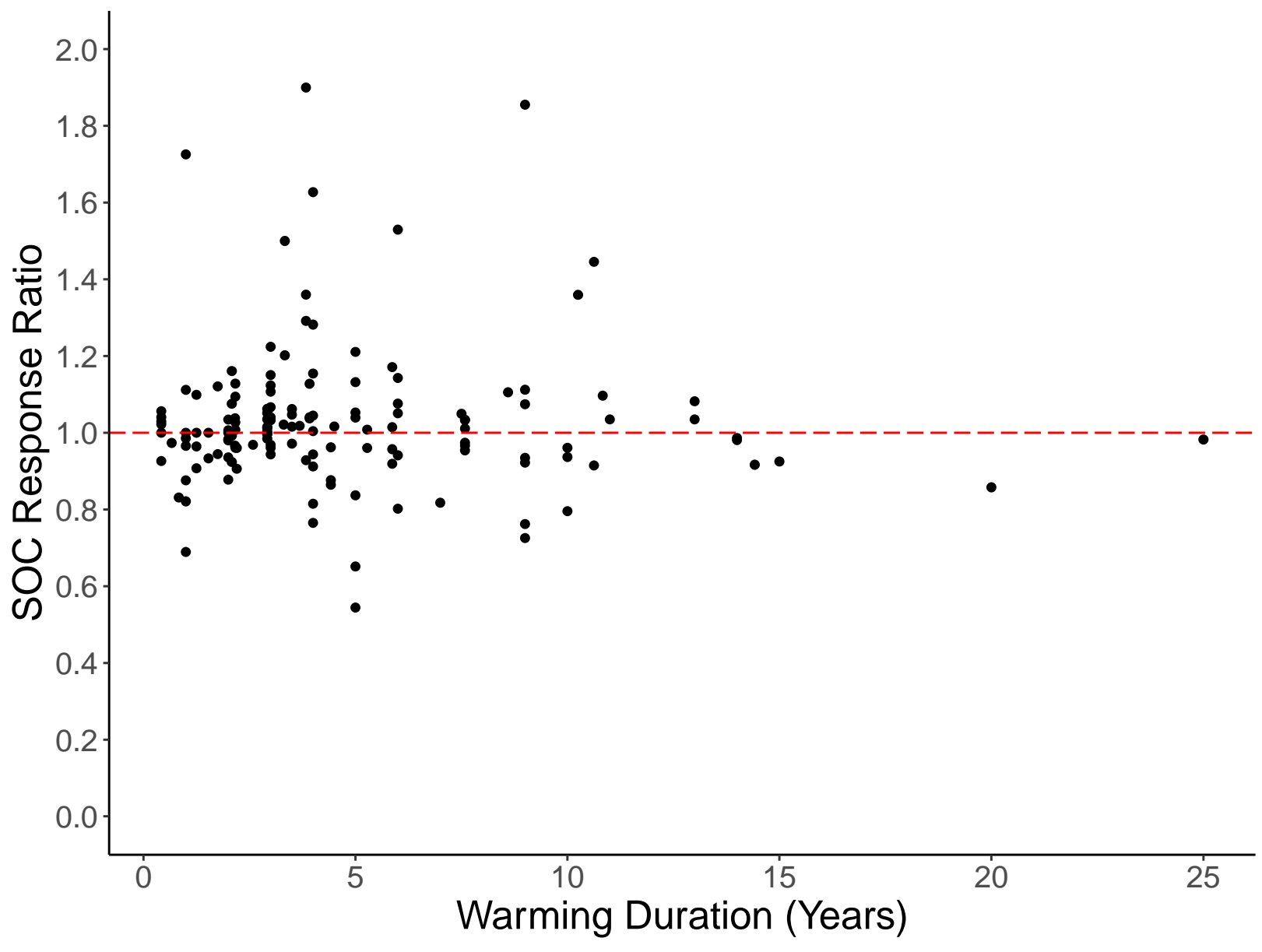


Figure S11: $95 \%$ credible areas for some (a) AWB and (b) CON parameters corresponding to pre-warming steady state $\mathrm{SOC}=50 \mathrm{mg} \mathrm{C} \mathrm{g}^{-1}$ soil, $\mathrm{DOC}=0.2 \mathrm{mg} \mathrm{C} \mathrm{g}^{-1}$ soil, $\mathrm{MIC}=2 \mathrm{mg} \mathrm{C} \mathrm{g}^{-1}$ soil, and $\mathrm{ENZ}=0.1 \mathrm{mg} \mathrm{C} \mathrm{g}^{-1}$ soil. Yellow shaded regions represent $80 \%$ credible areas and vertical purple lines indicate distribution mean. Note the deformity of the $E a_{S}$ and $E_{C_{\text {ref }}}$ densities. Parameter units are displayed in Supplemental Table 1.

(a)

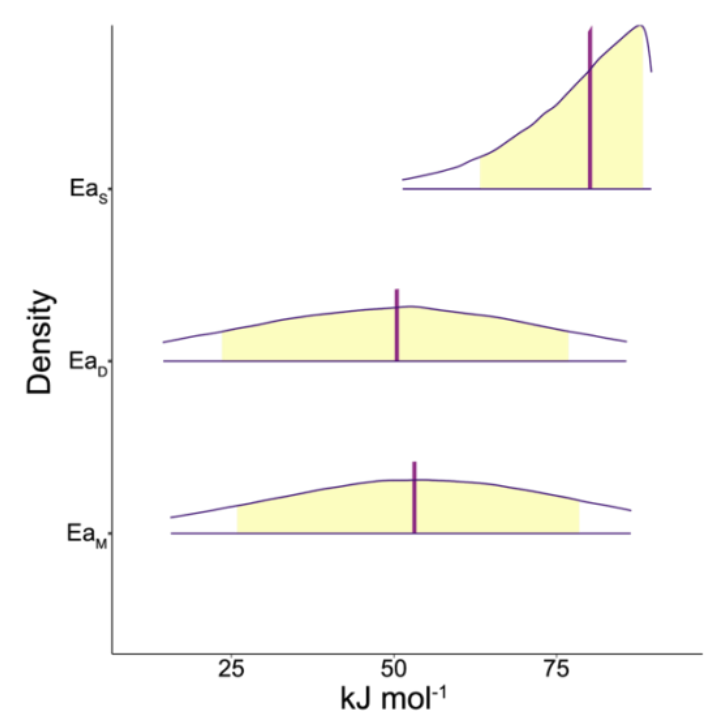

(b)

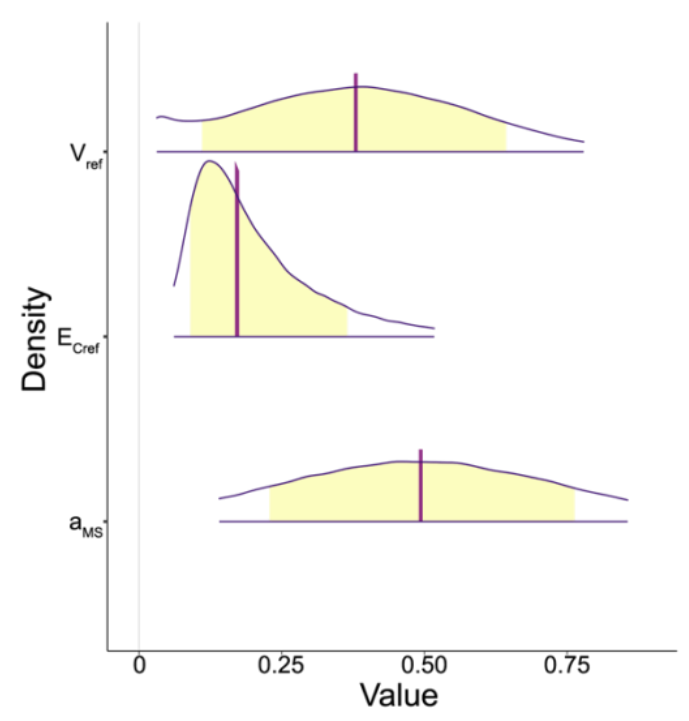

